\title{
Series: Cardiovascular outcome trials for diabetes drugs Lixisenatide and ELIXA
}

\author{
MILES FISHER
}

\begin{abstract}
ELIXA (Evaluation of Lixisenatide in Acute Coronary Syndrome) was an FDA mandated cardiovascular outcome trial with lixisenatide. In contrast to later cardiovascular outcome trials with glucagon-like peptide-1 (GLP-1) receptor agonists, it was performed in subjects with a recent myocardial infarction or hospitalisation for unstable angina within the previous 180 days. ELIXA compared lixisenatide and placebo in 6,068 subjects with type 2 diabetes and demonstrated noninferiority for major cardiovascular events plus unstable angina (cardiovascular death, myocardial infarction, stroke, unstable angina) but not superiority. Similarly, there was no difference in hospitalisation for heart failure which was a secondary outcome. A subsequent exploratory analysis showed that lixisenatide reduced progression of the urinary albumin to creatinine ratio in patients with macroalbuminuria, and was associated with a lower risk of new-onset macroalbuminuria. No clear clinical benefit has been established for lixisenatide, and there are alternative GLP-1 receptor agonists such as liraglutide, semaglutide and dulaglutide that are associated with a reduction in major adverse cardiovascular events. Br J Diabetes 2020;20:52-54
\end{abstract}

Key words: diabetes, cardiovascular outcome trial, lixisenatide

\section{Introduction}

Licensing requirements for new anti-diabetes drugs changed in the USA and Europe in 2008 and 2012, and a dedicated randomised controlled cardiovascular outcome trial (CVOT) was usually required either before or after licensing. ${ }^{1,2}$ This series describes and summarises the results of each of these CVOTs in the order in which they were published, describing the primary endpoint and important secondary outcomes from the principal publication, and directs attention to important subsequent publications of data from subgroups and post hoc analyses. ELIXA (Evaluation of Lixisenatide in

Address for correspondence: Professor Miles Fisher Department of Diabetes, Endocrinology \& Clinical Pharmacology, Glasgow Royal Infirmary, 84 Castle Street, Glasgow G4 OSF, UK

E-mail: miles.fisher@ggc.scot.nhs.uk

https://doi.org/10.15277/bjd.2020.241
Acute Coronary Syndrome) was the first published FDA-mandated cardiovascular outcome trial using a glucagon-like peptide-1 (GLP1) receptor agonist, ${ }^{3}$ after three previous negative trials with dipeptidyl peptidase-4 (DPP-4) inhibitors. ${ }^{4-6}$

\section{Background}

The GLP-1 receptor agonist lixisenatide was licensed for use in Europe in 2013 as a once daily injection. It was submitted by Sanofi at a similar time to the FDA in the USA. The FDA requested more information on cardiovascular safety than was available at that time from the phase III development programme. In particular, the FDA requested early interim results from the ELIXA trial. Sanofi decided that this approach could potentially compromise the integrity of the trial and withdrew the drug application. They re-submitted to the FDA when the results of ELIXA were available and lixisenatide was finally approved for use in the USA in 2015.

\section{ELIXA}

A paper describing the rationale, design and baseline characteristics of ELIXA was published in 2015. ${ }^{7}$ The primary endpoint was major adverse cardiovascular events plus hospitalisation for unstable angina (sometimes called 'MACE plus') comprising cardiovascular death, non-fatal myocardial infarction, non-fatal stoke, plus hospitalisation for unstable angina. Hospitalisation for heart failure was included as a secondary endpoint. The principal ELIXA results were presented in 2015 at the meeting of the American Diabetes Association (ADA) and published 6 months later in the New England Journal of Medicine. ${ }^{3}$ The design of the study and key baseline characteristics are described in Box 1. All subjects in ELIXA had a recent acute coronary syndrome so this was a secondary prevention study.

In ELIXA there was no significant difference in 'MACE plus', so non-inferiority was established but not superiority (Figure 1, Box 2). Rates of unstable angina were very low, and the frequency of unstable angina and hospitalisation for heart failure were similar in the two study groups. Lixisenatide was not associated with a higher rate of serious adverse events or severe hypoglycaemia, pancreatitis or pancreatic cancer.

\section{Other results from ELIXA}

Further publications from ELIXA are detailed in Box 2. Compared with other diabetes cardiovascular outcome trials, ${ }^{4-6,8,9}$ the number of further publications from ELIXA is small. The most important has 


\section{Box 1 Key features of ELIXA 3,7}

- ELIXA compared lixisenatide with placebo for a median of 25 months in 6,068 subjects

- Mean age of subjects was 60 years with a mean duration of diabetes of 10 years

- Mean baseline $\mathrm{HbA}_{1 \mathrm{c}}$ was $7.7 \%(61 \mathrm{mmol} / \mathrm{mol})$, and during the trial the average $\mathrm{HbA}_{1 \mathrm{c}}$ difference was $0.3 \%$

- $100 \%$ of subjects had an acute coronary syndrome within 180 days before screening, $22 \%$ had prior myocardial infarction before the qualifying event, $67 \%$ had prior percutaneous coronary intervention and $22 \%$ had investigator-reported heart failure at baseline

- The qualifying event was non-ST elevation myocardial infarction (NSTEMI) in 39\%, STEMI in 44\% and unstable angina in 17\%

- $66 \%$ of subjects were on metformin, $33 \%$ were on sulfonylureas, $2 \%$ were on thiazolidinediones and $39 \%$ were on insulin

Box 2 Results of the ELIXA trial

Principal result

- No reduction in 'MACE plus'3

Other results from ELIXA

- An exploratory analysis showed that lixisenatide reduced progression of the urinary albumin to creatinine ratio in patients with macroalbuminuria and was associated with a lower risk of new-onset macroalbuminuria ${ }^{10}$

- In ELIXA, increases in concentrations of natriuretic peptides (B-type natriuretic peptide (BNP), N-terminal prohormone BNP) occurred in the months preceding hospitalisation for heart failure ${ }^{18}$

- Baseline natriuretic peptides were powerful predictors of cardiovascular outcomes beyond heart failure and death, and were also predictive of myocardial infarction and stroke ${ }^{19}$

- A self-reported history of retinopathy and/or neuropathy at baseline and a longer duration of diabetes were markers for a high risk of recurrent cardiovascular events ${ }^{20}$

been a post-hoc exploratory analysis on renal outcomes, including by baseline albuminuria subgroup. For subjects with baseline macroalbuminuria, this was significantly reduced with lixisenatide at 2 years, and there was also a significant reduction in new onset macroalbuminuria. ${ }^{10}$ In subjects with baseline microalbuminuria, changes in the albumin to creatinine ratio did not reach statistical significance. No effects were seen in the decline in estimated glomerular filtration rate. The results are consistent with the effects of liraglutide and dulaglutide on renal outcomes in LEADER and REWIND. ${ }^{11,12}$

\section{Discussion}

ELIXA was the first published cardiovascular outcome trial for a GLP-1 receptor agonist and was negative. Subsequent outcome trials with liraglutide, ${ }^{9}$ semaglutide, albiglutide and dulaglutide ${ }^{13-15}$ have demonstrated significant reductions in major cardiovascular events, and the outcome trial with once-weekly exenatide did not demonstrate a clear benefit. ${ }^{16}$ Lixisenatide and exenatide are based on the exendin-4 peptide whereas the other molecules that have positive outcomes are true GLP-1 analogues, so different cardiovascular effects of the peptides might explain the negative ELIXA results. Lixisenatide is a short-acting receptor agonist with particular
Figure 1. 25-month incident rates (in \%) comparing lixisenatide and placebo for 'MACE plus', total mortality, cardiovascular (CV) mortality, non-fatal myocardial infarction, non-fatal stroke and hospitalisation for heart failure (HFH)
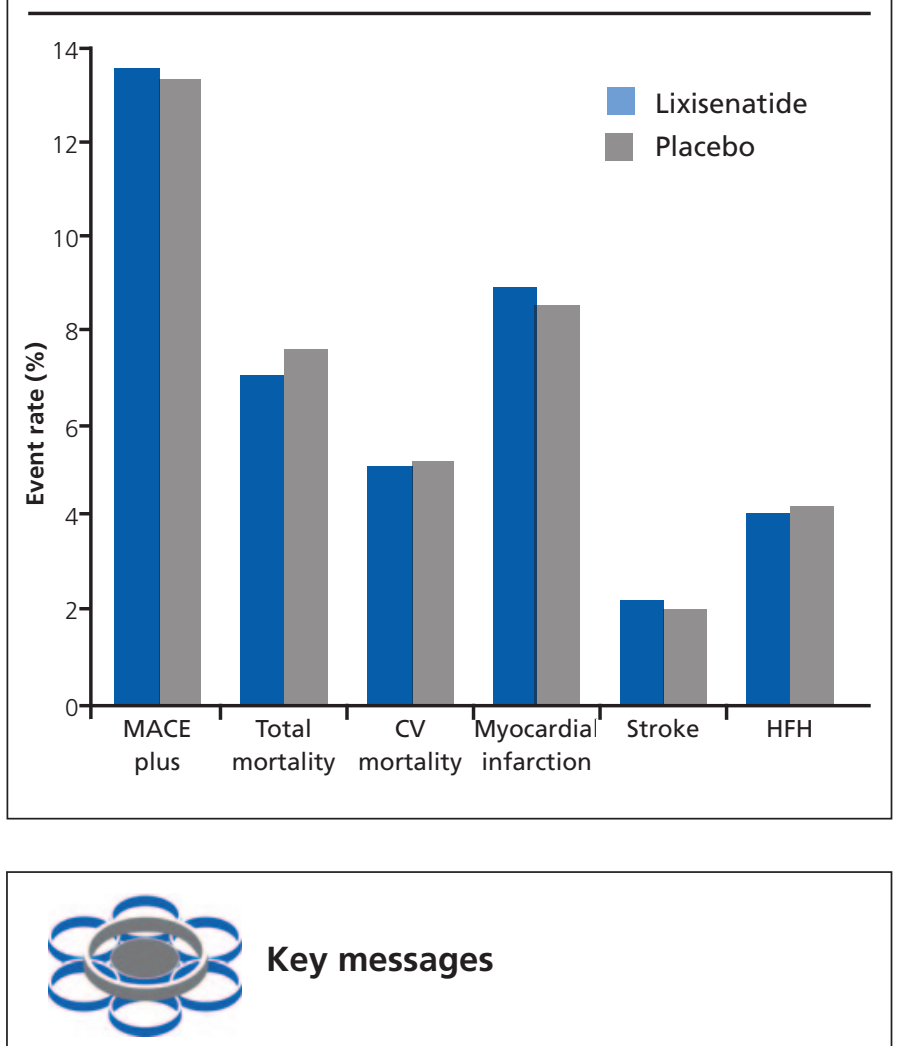

- ELIXA was the fourth published cardiovascular outcome trial of a diabetes drug, comparing lixisenatide and placebo

- In ELIXA, lixisenatide had no effect on atherosclerotic events of cardiovascular death, myocardial infarction or stroke, or on hospitalisation for heart failure

- Subsequent trials with liraglutide, subcutaneous semaglutide, albiglutide and dulaglutide demonstrated significant reductions in major adverse cardiovascular events

- It is uncertain if the negative results of ELIXA can be attributed to the patient group included (acute coronary syndromes), the intervention (lixisenatide), or both

effects on postprandial glucose. Meta-analysis has demonstrated that, compared with liraglutide and exenatide, lixisenatide showed a lower reduction in $\mathrm{HbA}_{1 \mathrm{c}}$ and body weight, so a lesser efficacy is also a possible explanation. ${ }^{17}$ Finally, ELIXA was performed in subjects with a recent acute coronary syndrome and the other positive studies were performed in subjects with stable atherosclerotic disease or high cardiovascular risk, and it is possible that GLP-1 receptor agonists are not beneficial in this specific subgroup of patients with cardiovascular disease. The results of ELIXA suggest the lixise- 
natide is the least useful GLP-1 receptor agonist due to its reduced efficacy and lack of cardiovascular protection.

Conflict of interest The author has received payment for advisory boards and/or lectures from AstraZeneca, Boehringer Ingelheim, Eli Lilly, GlaxoSmithKline, Janssen, MSD, NAPP, Novartis, Novo Nordisk, Sanofi, Takeda.

\section{Funding None.}

\section{References}

1. Food and Drug Administration. Guidance for industry. Diabetes mellitus - evaluating cardiovascular risk in new antidiabetic therapies to treat type 2 diabetes. Silver Spring, MD: US Department of Health and Human Services, Food and Drug Administration, Center for Drug Evaluation and Research (CDER), 2008. Available from: https://www. fda.gov/regulatory-information/search-fda-guidance-documents/diabetes-mellitus-evaluating-cardiovascular-risk-new-antidiabetic-therapiestreat-type-2-diabetes (accessed 23 Jan 2020)

2. European Medicines Agency. Guideline on clinical investigation of medicinal products in the treatment or prevention of diabetes mellitus. London: EMA, 2012. Available from: http://www.ema.europa.eu/docs/ en_GB/document_library/Scientific_guideline/2012/06/WC500129256. pdf (accessed 23 Jan 2020).

3. Pfeffer MA, Claggett $B$, Diaz $R$, et al, for the ELIXA Investigators. Lixisenatide in patients with type 2 diabetes and acute coronary syndrome. N Engl J Med 2015;373:2247-57. https://doi.org/10.1056/NEJMoa1509225

4. Scirica BM, Bhatt DL, Braunwald E, et al, for the SAVOR-TIMI 53 Steering Committee and Investigators. Saxagliptin and cardiovascular outcomes in patients with type 2 diabetes mellitus. N Eng/ J Med 2013;369:131726. https://doi.org/10.1056/NEJMoa1307684

5. White WB, Cannon CP, Heller SR, et al, for the EXAMINE Investigators. Alogliptin after acute coronary syndrome in patients with type 2 diabetes. N Engl J Med 2013;369:1327-35. https://doi.org/10.1056/NEJMoa1305889

6. Green JB, Bethel A, Armstrong PW, et al, for the TECOS Study Group. Effect of sitagliptin on cardiovascular outcomes in type 2 diabetes. $N$ Engl J Med 2015;373:232-42. https://doi.org/10.1056/NEJMoa1501352

7. Bentley-Lewis R, Aguilar D, Riddle MC, et al. Rationale, design, and baseline characteristics in Evaluation of LIXisenstide in Acute Coronary Syndrome, a long-term cardiovascular end point trial of lixisenatide versus placebo. Am Heart J 2015;169:631-8 https://doi.org/10.1016/j.ahj.2015.02.002

8. Zinman B, Wanner C, Lachin JM, et al, for the EMPA-REG OUTCOME investigators. Empagliflozin, cardiovascular outcomes, and mortality in type 2 diabetes. N Engl J Med 2015;373:2117-28. https://doi.org/10.1056/NEJMoa1504720

9. Marso SP, Daniels GH, Brown-Frandsen K, et al, for the LEADER steering committee on behalf of the LEADER Trial Investigators. Liraglutide and cardiovascular outcomes in type 2 diabetes. N Engl J Med 2016; 375:311-22. https://doi.org/10.1056/NEJMoa1603827

10. Muskiet MHA, Tonneijck L, Huang $Y$, et al. Lixisenatide and renal outcomes in patients with type 2 diabetes and acute coronary syndrome: an exploratory analysis of the ELIXA randomised, placebo-controlled trial. Lancet Diabetes Endocrinol 2018;6:859-69. https://doi.org/10.1016/S2213-8587(18)30268-7

11. Mann JFE, Orsted DD, Brown-Frandsen $K$, et al, for the LEADER Steering Committee and Investigators. Liraglutide and renal outcomes in type 2 diabetes. N Engl J Med 2017;377:839-48. https://doi.org/10.1056/NEJMoa1616011

12. Gertsein HC, Colhoun HM, Dagenais GR, et al. Dulaglutide and renal outcomes in type 2 diabetes: an exploratory analysis of the REWIND randomised, placebo-controlled trial. Lancet 2019;394:131-8. https://doi.org/10.1016/S0140-6736(19)31150-X

13. Marso SP, Bain SC, Consoli A, et al, for the SUSTAIN-6 Investigators Semaglutide and cardiovascular outcomes in patients with type 2 diabetes. N Engl J Med 2016;375:1834-44. https://doi.org/10.1056/NEJMoa1607141

14. Hernandez AF, Green JB, Janmohamed S, et al, for the Harmony Outcomes Committees and Investigators. Albiglutide and cardiovascular outcomes in people with type 2 diabetes and cardiovascular disease (Harmony Outcomes): a double-blind, randomised, placebo-controlled trial. Lancet 2018;392:1519-29. https://doi.org/10.1016/S0140-6736(18)32261-X

15. Gertsein HC, Colhoun HM, Dagenais GR, et al. Dulaglutide and cardiovascular outcomes in type 2 diabetes (REWIND): a double-blind, randomised, placebo-controlled trial. Lancet 2019;394:121-30. https://doi.org/10.1016/S0140-6736(19)31149-3

16. Holman RR, Bethel MA, Mentz RJ, et al, for the EXSCEL Study Group. Effects of once-weekly exenatide on cardiovascular outcomes in type 2 diabetes. N Engl J Med 2017;377:1228-39. https://doi.org/10.1056/NEJMoa1612917

17. Schmidt LJ, Habacher W, Augustin T, et al. A systematic review and meta-analysis of the efficacy of lixisenatide in the treatment of patients with type 2 diabetes. Diabetes Obes Metab 2014;16:769-79. https://doi.org/10.1111/dom.12269

18. Wolsk $E$, Claggett $B$, Diaz $R$, et al. Increases in natriuretic peptides precede heart failure hospitalization in patients with a recent coronary event and type 2 diabetes. Circulation 2017:136:1560-2. https://doi.org/10.1161/CIRCULATIONAHA.117.029503

19. Wolsk E, Claggett B, Pfeffer MA, et al. Role of B-type natriuretic peptide and $\mathrm{N}$-terminal prohormone BNP as predictors of cardiovascular morbidity and mortality in patients with a recent coronary event and type 2 diabetes mellitus. J Am Heart Assoc 2017 May 29;6(6). pii: e004743. https://doi.org/10.1161/JAHA.116.004743

20. Seferovic JP, Bentley-Lewis R, Claggett B, et al. Retinopathy, neuropathy, and subsequent cardiovascular events in patients with type 2 diabetes and acute coronary syndrome in the ELIXA: the importance of disease duration. J Diabetes Res 2018 Dec 16:2018:1631263. https://doi.org/10.1155/2018/1631263 